\title{
Generation Times of Bacteria: Real and Artificial Distributions
}

\author{
By E. O. POWELL \\ Microbiological Research Establishment, Porton Down, Salisbury, Wiltshire
}

(Accepted for publication 27 June 1969)

Under the subheading 'Real and artificial distributions' I gave in a previous paper (Powell, 1964) a brief sketch of the relationships between the various distributions which come to be considered in any discussion of the pattern of bacterial generation times. The present note is an elaboration of this sketch, prompted by Painter \& Marr's (1967) imputation of error in my treatment. It will be seen that the supposed discrepancies arise from nothing more than a misunderstanding of symbols and terminology.

If individual generation times in a growing culture were independent of one another there would be no problem; in fact growth is affected by internal correlations. Here I shall deal with the only manageable case which is at all realistic: significant correlation between generation times of mother and daughter organisms, other correlations being irrelevant or quantitatively negligible. It will be convenient to think of the culture as maintained in a steady state in a chemostat (exactly the same relationships are obtained by considering exponential growth in batch culture, but the argument is more tedious). Those organisms which remain in the culture until their termination, i.e. those whose generation times compose the carrier distribution, I shall call 'successful' organisms.

\section{The artificial distributions}

If we have a hypothetical mechanism from which precise inferences to the pattern of generation times $(\tau)$ can be made, we are naturally led to construct, for comparison with experiment, a frequency function (say $f(\tau)$ ) applicable in some sense to all organisms whether considered as mothers or daughters; and a bivariate function (say $h(\xi, \tau)$ ) for the joint distribution of mother $(\xi)$ and daughter $(\tau)$ generation times, with the property that its border distributions are alike of the form $f(\tau)$ :

$$
\int_{0}^{\infty} h(\xi, \tau) d \xi=f(\tau) ; \quad \int_{0}^{\infty} h(\xi, \tau) d \tau=f(\xi) .
$$

This construction implies, then, the assumption that mothers and daughters of the same generation time have the same frequency. This is not true of actual growth and so it must be admitted at once that $f(\tau)$ and $h(\xi, \tau)$ are artificial in the sense that they are not directly represented in the growing culture. If in the chemostat the population were maintained constant by selecting organisms for removal in a manner independent of their generation times, $f(\tau)$ and $h(\xi, \tau)$ would be realized; in fact, the probability that an organism of generation time $\tau$ is successful is $e^{-\nu \tau}$ (where $\nu$ is the growth rate) and so the probability is greater the smaller is $\tau$. Suppose that during a short interval in the life of the culture the newly-formed organisms had the distribution $f(\tau)$; of these the successful organisms would have predominantly short generation times. Because of the association between successive generation times, the progeny of the successful organisms would not (in general) have the distribution $f(\tau)$. 
Thus the composition of the population would alter. If we write $\mathscr{P}(\tau)$ for the 'population distribution', i.e. the generation time distribution of all those organisms, whether successful or not, whose inception occurs in the culture vessel, the carrier distribution $(\mathscr{C}(\tau)$, corresponding to the successful organisms) must be such that both $\mathscr{C}$ and $\mathscr{P}$ constantly reproduce themselves:

$$
\mathscr{C}(\tau) \equiv 2 e^{-\nu \tau \mathscr{P}}(\tau)
$$

the factor 2 arising because on average only one of each pair of sisters is successful. The distribution $f(\tau)$ cannot reproduce itself under the actual circumstances of growth, but in fact it determines $\mathscr{P}(\tau)$ through the integral equation (Powell, 1964):

$$
\mathscr{P}(\tau)=2 \int_{0} e^{-\nu \xi} \frac{h(\xi, \tau)}{f(\xi)} \mathscr{P}(\xi) d \xi .
$$

This equation at the same time determines the growth rate because its only solution is $\mathscr{P}(\tau) \equiv 0$ unless $\nu$ is such that 2 is a characteristic number of the kernel

$$
e^{-\nu \xi} h(\xi, \tau) \mid f(\xi) \text {. }
$$

Equation (2) may be reached by an approach rather different from my earlier one. Given a successful organism of generation time $\xi$, we can say that the probability of some one of its daughters having generation time $\tau$ is unaffected by the circumstances of growth; that is, the distribution of $\tau$ for any fixed $\xi$ is unaffected. To determine $\mathscr{P}$ and $\mathscr{C}$ we therefore seek a variable multiplier $\lambda(\xi)$ such that

$$
\begin{gathered}
\mathscr{C}(\xi)=\lambda(\xi) f(\xi)=\lambda(\xi) \int_{0}^{\infty} h(\xi, \tau) d \tau, \\
\mathscr{P}(\tau)=\int_{0}^{\infty} \lambda(\xi) h(\xi, \tau) d \xi,
\end{gathered}
$$

together with the condition (I) imposed on $\mathscr{C}$ and $\mathscr{P}$ by the washing out of organisms from the culture.

Then

and

$$
\lambda(\xi) \equiv \mathscr{C}(\xi) \mid f(\xi)=2 e^{-\nu \xi \mathscr{P}(\xi) / f(\xi)}
$$

as before.

$$
\mathscr{P}(\tau)=2 \int_{0}^{\infty} e^{-\nu \xi} \frac{h(\xi, \tau)}{f(\xi)} \mathscr{P}(\xi) d \xi
$$

The construction of the frequency functions $f$ and $h$ has some slight historical sanction and mathematically is usually relatively easy to do. In experimental work, care has to be taken to obtain a sample of generation times which is unbiased with respect to $f$ or $h$ (Powell, I964); an attempt to sample for $\mathscr{C}$ or $\mathscr{P}$ needs equal care, however, and for a given sample size is more time-consuming.

It is the function $f(\tau)$ which I call 'the generation time distribution' tout court.

\section{The real distributions}

If it were conveniently possible to measure the generation times associated with a random selection of fissions occurring in a steady-state continuous culture, they would provide a sample from the realized joint distribution of mother and daughter generation times, say $\mathscr{H}(\xi, \tau)$, with borders $\mathscr{C}(\xi)$ (corresponding to mothers) and $\mathscr{P}(\tau)$ (corresponding to daughters). 
We now approach from the opposite direction the relation between $f$ and $h$ on the one hand and $\mathscr{C}, \mathscr{P}$ and $\mathscr{H}$ on the other. We ask if it is possible to construct from $\mathscr{H}(\xi, \tau)$ an associated bivariate distribution whose borders are alike. As before, we note that for any fixed $\xi$, the distribution of $\tau$ is not altered by the circumstances of growth, and in fact we have for every $\xi$

$$
\frac{h(\xi, \tau)}{f(\xi)} \equiv \frac{\mathscr{H}(\xi, \tau)}{\mathscr{C}(\xi)}
$$

identically in $\tau$; each member of (3) is the frequency function of the $\tau$ associated with a particular $\xi$. We therefore seek a variable multiplier $\kappa(\xi)$ such that

with the further condition

$$
\begin{gathered}
\kappa(\xi) \int_{0}^{\infty} \mathscr{H}(\xi, \tau) d \tau=\kappa(\xi) \mathscr{C}(\xi)=f_{a}(\xi), \text { say: } \\
\int_{0}^{\infty} \kappa(\xi) \mathscr{H}(\xi, \tau) d \xi=f_{a}(\tau)
\end{gathered}
$$

$$
\int_{0}^{\infty} f_{a}(\tau) d \tau=\mathrm{I}
$$

That is, we try to satisfy the integral equation

or

$$
\kappa(\tau) \mathscr{C}(\tau)=\int_{0}^{\infty} \kappa(\xi) \mathscr{H}(\xi, \tau) d \xi
$$

$$
f_{a}(\tau)=\int_{0}^{\infty} f_{a}(\zeta) \frac{\mathscr{H}(\xi, \tau)}{\mathscr{C}(\xi)} d \xi
$$

It is easy to verify that the Fredholm determinant of the kernel in (4) vanishes in virtue of the relation

$$
\mathscr{C}(\xi)=\int_{0}^{\infty} \mathscr{H}(\xi, \tau) d \tau
$$

(see, e.g. Aitken, 1942), and so (4) always has a non-trivial solution (see, e.g. Smithies, I958). We readily identify this solution by substituting (3) into (4):

which is satisfied by

$$
f_{a}(\tau) \equiv \int_{0}^{\infty} f_{a}(\xi) \frac{h(\xi, \tau)}{f(\xi)} d \xi
$$

because

$$
f_{a}(\tau) \equiv f(\tau)
$$

$$
\int_{0}^{\infty} h(\xi, \tau) d \xi=f(\tau)
$$

Conversely, if in (2) we substitute (3) we obtain

$$
\mathscr{P}(\tau)=\int_{0}^{\infty} \mathscr{H}(\xi, \tau) d \xi
$$

which is also true.

The frequency functions $f$ and $h$ can thus be completely reconciled with $\mathscr{C}, \mathscr{P}$ and $\mathscr{H}$. 


\section{Painter and Marr's analysis}

Painter \& Marr (1967) showed that there is associated with a culture in steady growth a frequency function of generation times (say, temporarily, $f_{p m}(\tau)$ ) which does reproduce itself under the actual circumstances of growth, that

and that

$$
\mathscr{C}(\tau)=2 e^{-\nu \tau} f_{p m}(\tau)
$$

$$
2 \int_{0}^{\infty} e^{-\nu \tau} f_{p m}(\tau)=\mathrm{I} \text {. }
$$

They show further that the mean of this distribution is greater than $(\log 2) / \nu$. Their reasoning is so far correct. But unfortunately they use the symbol $f(\tau)$ for the distribution in question, and then identify it with the $f(\tau)$ discussed above and used by me elsewhere in cases in which mother and daughter generation times are not independent. They infer that $f(\tau)$ in the latter sense cannot be symmetrical and that its mean cannot be $(\log 2) / \nu$ (as it is under the restricted Koch \& Schaechter (I962) hypothesis; Powell, 1964).

It should now be clear that Painter \& Marr's distribution is identical with $\mathscr{P}(\tau)$, which I have called the 'population distribution' and which is to be carefully distinguished from $f(\tau)$. There is, then, no real discrepancy. $\mathscr{P}$ relates to all the organisms in the culture, past, present and to come. The distribution for those organisms extant at any one time is

$$
\mathscr{I}(\tau)=2\left(\mathrm{I}-e^{-\nu \tau}\right) \mathscr{P}(\tau)
$$

When mother and daughter generation times are not independent, ' $\mathscr{P}(\tau)$ replaces $f(\tau)$ in expressions for the carrier and age distributions and for $\mathscr{I}(\tau)$ ' (Powell, 1964).

\section{Conclusion}

The difference between $\mathscr{P}(\tau)$ and $f(\tau)$ is usually not great, and as the extreme examples in Powell (I956) show, an error of less than $3 \%$ is incurred if $\nu$ is calculated from the $\tau$ as if they were independent. Although I have written of $f(\tau)$ as 'artificial', an effective hypothesis about the distribution of $\tau$ leads directly to it; yet there is something adventitious about $\mathscr{P}(\tau)$ in that it comes to differ from $f(\tau)$ only as a secondary result of multiplicative growth.

I think therefore that both distributions still have a place in the theory of the pattern of generation times. But their precise meanings must be kept clearly in mind.

\section{REFERENCES}

Artken, A. C. (1942). Determinants and Matrices. 2nd ed. Edinburgh: Oliver and Boyd.

Koch, A. L. \& SchAeCHTER, M. (1962). A model for statistics of the cell division process. J. gen. Microbiol. 29, 435.

Painter, P. R. \& MARR, A. G. (1967). Inequality of mean interdivision time and doubling time. J. gen. Microbiol. 48, 155 .

Powell, E. O. (1956). Growth rate and generation time of bacteria, with special reference to continuous culture. J. gen. Microbiol. $15,492$.

Powell, E. O. (1964). A note on Koch \& Schaechter's hypothesis about growth and fission of bacteria. J. gen. Microbiol. 37, 23 I.

SMITHIES, F. E. (1958). Integral Equations. Cambridge University Press. 